\title{
THE DAWN OF ROBO-NANO NEUROSURGERY
}

\author{
Krešimir Rotim
}

\begin{abstract}
Clinical Department of Neurosurgery, Sestre milosrdnice University Hospital Center, Zagreb, Croatia
\end{abstract}
Although it is one of the youngest fields of medicine, neurosurgery seems to be around for centuries. According to archeological evidence, surgery on the skull is over 10,000 years old ${ }^{1}$. In the ancient Egypt, neurosurgery was performed 2000 years BC, as shown in the Edwin Smith Papyrus².

In the $19^{\text {th }}$ century, neurosurgery was associated with vast mortality. It was during the $20^{\text {th }}$ century that neurosurgery has achieved important improvements to both technique and diagnosis.

Neurosurgery is the field of medicine where advanced technology feels at home, adapting new techniques and devices in an effort to increase the efficacy and safety of brain and spine surgery. Among these recent advances are surgical robotics and nanotechnology.

The use of a mechanical device, automated or with remote control, in order to manipulate the instruments directly in contact with the patient represents a relatively new procedure ${ }^{3}$. Robotic technology has been incorporated into stereotactic and endoscopic procedures and is used in robotized microscope, telepresence, and tumor resection ${ }^{1,2,4-6}$.

Technically, surgical robots can be divided into passive and active systems. A passive system is the one in which the surgeon provides physical energy to drive the surgical tool ${ }^{7}$. An active robotic system is the one in which the robot actively interacts with the patient, allowing more complicated motions and the surgeon has the ability to supervise and intervene when necessary ${ }^{8}$.

Surgical robots can also be classified according to how the surgeon interacts with them. They are divided into supervisory-controlled system, telesurgical system, and shared-control system.

Since Kwoh et al. attempted a robotic brain biopsy in the late 1980s, interest in this field and its potential clinical benefits has grown ${ }^{8}$. Neurosurgery has defined concepts of manual microsurgical techniques and these are already embedded effectively and successfully in standard practice. Therefore, integration of surgical robotics represents an interesting dilemma. Although its theoretical advantages seem most suited to neurosurgical disease, the state of the art has not yet matched the theory ${ }^{9}$.

Several robotic solutions have been developed to address the specific challenges associated with interventions on the brain ${ }^{10,11}$. The NeuroMate robotic surgical system was the first Food and Drug Administration (FDA) approved robotic device for neurosurgery ${ }^{10}$. The Minerva neurosurgical robot followed. Both systems involve a passive robotic arm which moves in a preprogrammed direction to a specific site defined by integrated neuronavigation systems for stereotactic biopsy or functional neurosurgical applications ${ }^{12}$.

Several robotic systems have been developed to address the challenges encountered in spinal surgery. As with brain applications, these devices are enhanced significantly by advances in intraoperative image guidance. In general, research in this area has focused on accurate placement of spinal instrumentation, citing the theoretically increased accuracy that robotics offers ${ }^{13}$. In radiosurgery, robotic solutions to spine motion with respiration have also been extremely usefu $1^{14}$. As with intracranial radiosurgical applications, the most common robotic subtype in spinal stereotactic radiosurgery is the supervisory-controlled system. The Cyberknife relies on a predetermined plan which targets spinal pathology for focused beam radiotherapy. Using feedback mechanisms, this system can adjust its trajectory to correct for patient movement, most of which result from respiration. This use of robotics has also been expanded to intracranial use, given the pos- 
sibility of brain shift. A recent addition to the $\mathrm{Cy}-$ berknife system is the RoboCouch Patient Positioning System (Accuracy), which uses similar technology to reposition the patient during the course of treatment.

In the promising field of robotic neurosurgery, special attention must be paid to the brain and spine surgical anatomy. Integrations of new focused technologies can be adapted more easily into highly specialized operating environment. The robots most widely used in neurosurgery have been the products of this contextual research concentrated on the central nervous system-specific solutions. Attempts to adapt other instrumentation for neurosurgical use have proven to be less effective ${ }^{9}$.

Nanotechnology defines the science and engineering providing entirely new tools and capabilities, concerned with the design, synthesis, characterization and application of materials and devices that have functional organization in at least one dimension on the nanometer (one billionth of meter) capable to interact with cells and tissues at the molecular-subcellular level with a high degree of functional specificity, involving innumerable aspects of technology, including neurosurgery too ${ }^{15}$.

The practical use of nanotechnology in the field of neurosurgery could be by way of nano-manipulation, nano-imaging and nano-neuromodulation. Nano-manipulation refers to techniques designed to perform 'surgery' on the nervous system. Nano-imaging refers to capabilities to view the nervous system at the cellular or subcellular level. Nano-neuromodulation includes the possibility to control pain, in a fashion of 'biologic pumps', used both in neuropathic and chronic pains ${ }^{16}$.

Nanotechnology offers a highly interdisciplinary area of research in conjunction with basic and clinical neuroscience advances: molecular biology, neurophysiology and neuropathology of the nervous system ${ }^{15}$.

Neurosurgeons of the present and future must take an active function in shaping the plan and research to ensure maximal clinical relevance and patient benefit, to provide or administer the adequate technology or medication to the site where it is needed in the central nervous system. I think nanoneurosurgery and nanobioelectronics (integration of nanotechnology, device, imaging, AI, supercomputing, genomics and cellular therapeutics) will change the entire field of neurosurgery in the next 5-10 years.

\section{References}

1. Louw DF, Fielding T, McBeth PB, Gregoris D, Newhook P, Sutherland GR. Surgical robotics: a review and neurosurgical prototype development. Neurosurgery. 2004;54(3):525-37. doi: 10.1227/01.NEU.0000108638.05274.E9

2. Mc Beth PB, Louw DF, Rizun PR, Sutherland GR. Robotics in neurosurgery. Am J Surg. 2004;188(4):68-75. doi: 10.1016/j. amjsurg.2004.08.004

3. Nathoo N, Cavusoglu MC, Vogelbaum MA, et al. In touch with robotics: neurosurgery for the future. Neurosurgery. 2005; 56:421-33. doi: 10.1227/01.NEU.0000153929.68024.CF

4. Giorgi C, Eisenberg H, Costi G, Gallo E, Garibotto G, Casolina DS. Robot-assisted microscope for neurosurgery. J Image Guid Surg. 1995;1(3):158-63. doi: 10.3109/1092908950910 5690

5. Hongo K, Goto T, Kakizawa Y, Koyama J, Kawai T, Kan K, et al. Micromanipulator system (NeuRobot): clinical application in neurosurgery. Int Congr Ser. 2003;1256:509-13. doi: 10.1016/S0531-5131(03)00247-4

6. Benabid AL, Lavallee S, Hoffmann D, Cinquin P, Demongeot J, Danel F. Potential use of robots in endoscopic neurosurgery. Acta Neurochir Suppl (Wien). 1992;54:93-7.

7. Mckay-Davies I, Bann S, Darzi A. Robotics in surgery. Student BMJ. 2002;10:215-58.

8. Kwoh YS, Hou J, Jonckheere EA, Hayati S. A robot with improved absolute positioning accuracy for $\mathrm{CT}$ guided stereotactic brain surgery. IEEE Trans Biomed Eng. 1988;35(2):153-60. doi: $10.1109 / 10.1354$

9. Karas CS, Chiocca EA. Neurosurgical robotics: a review of brain and spine applications. J Robotic Surg. 2007;1:39-43. doi: 10.1007/s11701-006-0006-6

10. Benabid AL, Cinquin P, Lavelle $\mathrm{S}$, et al. Computer driven robot for stereotactic surgery connected to CT scan, and magnetic resonance imaging: technological design and preliminary results. Appl Neurophysiol, 1987;50:153-4. doi: 10.1159/000100701

11. Drake JM, Joy M, Goldberg A, et al. Computer and robot-assisted resection of thalamic astrocytomas in children. Neurosurgery. 1991;29:27-31. doi: 10.1097/00006123-199107000-00005

12. Glauser D, Frankhauser H, Epitaux M, et al. Neurosurgical robot Minerva: first results and current developments. J Image Guid Surg. 1995;1:266-72. doi: 10.3109/10929089509106332

13. Goto T, Hongo K, Kakizawa Y, et al. Clinical application of robotic telemanipulator system in neurosurgery. J Neurosurg. 2003;99:1082-4. doi: 10.3171/jns.2003.99.6.1082

14. Lieberman IH, Togawa D, Kayanja MM, et al. Bone-mounted miniature robotic guidance for pedicle screw and translaminar facet screw placement: Part 1. Technical development and a test case result. Neurosurgery. 2006;59:641-8.

15. Russell JA. Nanotechnology and neurosurgery. J Nanosci Nanotechnol. 2009;9:5008-13. doi: 10.1166/jnn.2009.GR03

16. Saini R, Saini S. Nanotechnology and surgical neurology. Surg Neurol Int. 2010;1:57. doi: 10.4103/2152-7806.69384 